\title{
Transient Paralytic Attacks of Obscure Nature: The Question of Non-Convulsive Seizure Paralysis
}

\author{
C. MILLER FISHER
}

SUMMARY: Eleven patients with transient paralytic attacks of obscure nature are described. Paralysis could involve face or leg alone, face and hand, or face, arm and leg. The duration varied from two minutes to one day. Four patients had brain tumors, six probably had brain infarcts, and one a degenerative process. The differential diagnosis included TIAs, migraine accompaniments, and seizures. In the absence of good evidence for the first

RESUMÉ: Nous décrivons onze patients présentant des attaques paralytiques transitoires de nature obscure. La paralysie peut impliquer la face ou la jambe seulement, la face et la main, ou la face, le bras ou la jambe. La durée variait de 2 minutes à un jour. 4 patients souffraient de tumeur cérébrale, six d'infarctus cérébraux et un d'un processus dégénératif. Le diagnostic différentiel incluait des ischémies transitoires, la migraine accompagnée et l'épilepsie. Devant l'absence d'évidence con- two, the cases are discussed from the standpoint of possibly representing nonconvulsive seizure paralysis (ictal paralysis, inhibitory seizure paralysis or somatic inhibitory seizure). Because of the difficulty in defining seizures as well as TIAs and migraine in their atypical variations, a firm conclusion concerning the mechanisms of the spells was not attained. Two cases of the hypertensive amaurosis-seizure syndrome have been added as further examples of ictal deficits.

vaincante en faveur des deux premiers diagnostics, nous discutons de la présence possible de paralysie par crises non. convulsives (paralysie ictale, paralysie par crise inhibitrice ou crise somatique inhibitrice). Parce qu'il est aussi difficile de définir "une crise" que de définir la migraine ou les ischémies transitoires dans leurs variantes atypiques, nous n'avons pu tirer de conclusion ferme. Nous décrivons aussi deux cas de crise amaurotique hypertensive.
Reprint requests to Dr. C. Miller Fisher, Dept. of Neurology, Harvard Medical School, Massachusetts General Hospital, Fruit St., Boston 02114 U.S.A.

From the Neurological Service of the Massachusetts General Hospital and the Department of Neurology, Harvard Medical School, Boston, Massachusetts

This study was supported in part by NINCDS, Grant NSOS152
In cerebrovascular disease, recognition of transient attacks of cerebral ischemia (TIAs) is of practical importance in permitting therapy which forestalls a disastrous thrombotic stroke. Often this requires the differentiation of TIAs from noncerebrovascular spells of various kinds - cerebral seizures, migraine, Meniere's disease, cardiac syncope, etc. - a task which is usually straightforward. Occasionally, however, atypical spells are met which seem to fall outside recognized categories and on the Stroke Service of the Massachusetts General Hospital we have examined a series of patients with transient spells of focal weakness of obscure nature. These cases are the subject of the present report.

In some of our cases an unrecognized vascular mechanism could be responsible while in other cases atypical migraine is a possibility. In late-life migraine accompaniments, the two most reliable clinical clues are the presence of visual phenomena with a "build-up" and/or the occurrence of a sensory march. A headache may or may not be present. Our cases, however, did not seem to be vascular or migrainous and we have been led to consider the possibility of nonconvulsive seizure paralysis, a rare diagnosis whose very existence has been difficult to establish. In the International Classification of Epileptic Seizures, the diagnosis is followed by a question mark (?), the only type of seizure so designated in the entire classification (Gastaut, 1970). Our cases will therefore be presented mainly from the standpoint of feasability or likelihood of non-convulsive seizure paralysis.

The vagueness surrounding the phenomenon which is variously termed 
inhibitory seizure paralysis, somatic inhibitory seizure, and ictal paralysis is due to the obvious difficulty of defining a paralyzing seizure in the absence of clonic or tonic movements. Documentation in the literature has usually been based on patient reports rather than eyewitness accounts, and minor twitching may have been overlooked not only by the patient but by witnesses as well. In some of our cases, it was possible that subtle convulsive activity was missed.

Jackson (1890) wrote that in some cases of epileptiform seizures the patient said his hand "falls dead", when there were no spasms in it while the face on the same side was convulsing. He thought that a slight discharge might cause after-exhaustion of some elements without producing an actual convulsion. In Dutil's (1883) Case 6 the subject tied a handkerchief around the thigh to arrest an ascending focal seizure starting in the right foot, nonetheless, the right arm became paralyzed a few moments later and aphasia slowly appeared 30 minutes later, lasting about 12 hours. Gowers (1885) thought that such paralysis was apt to follow a local sensory attack in the limbs. Holmes' (1927) patient had recurrent focal motor seizures beginning in the fingers, but sometimes he suddenly lost power in the hand for a few minutes without convulsive movements of any kind. Collier (1928) stated that "simple paralysis ... may be the sole manifestation of an epileptic attack just as the paralysis of migraine usually is the sole manifestation of the migraine attack in which it occurs." He referred to two epileptic patients who had many sudden attacks of local transient paralysis and occasionally the accompaniment of an aura which revealed their real nature. In another case, continual focal motor seizures involving the right upper extremity were followed by temporary paralysis of the face and leg on the right side, although they had not participated in the convulsion. Penfield and Jasper (1954) described a case in which for one week postoperatively, convulsive movements involved the left face and the eyes deviated to the right following which the left arm became paralyzed and the left leg weak, although neither showed convulsive movements. Here, brain edema may have been a factor. Lennox (1960) described a child who had akinetic spells of unconsciousness followed by weakness of the right arm for 15 minutes up to several hours. Efron's (1961) patient could, by means of rubbing his leg with a brush, partially arrest sensory seizures beginning in the toes of the left foot. The left arm, although not involved by the seizure, became so weak for up to 12 hours after an attack that the patient could scarcely hold anything in the hand. In the case of Kofman and Tasker (1967), a marching numbness spread over the left side of the body, face, arm and leg, and the limbs became transiently weak, although they did not convulse. Rasmussen (1974) referred to somatic inhibitory seizures arising in the central region or in the supplementary motor area and in which paralysis preceded tonic or clonic movement by a few seconds.

Criteria for establishing the diagnosis of non-convulsive seizure paralysis might be arranged according to reliability in the following descending order: (1) The development of focal paralysis seconds or minutes before convulsive movements begin in the limb. This particular event is probably the best evidence that the phenomenon of ictal paralysis exists. (2) The occurrence, sometimes without a convulsion, of a deficit similar in every way to the post-ictal deficit which follows other seizures associated with a convulsion; (3) the occurrence in one limb of a convulsive seizure and at the same time the development of paralysis in another limb without convulsive movements; (4) the occurrence in some paralytic spells of an aura which precedes other epileptic seizures; (5) a seizure discharge in the electroencephalogram (EEG) during an episode of paralysis; (6) episodes of paralysis with or without accompanying sensory phenomena in a clinical situation where a seizure rather than another type of episode is to be expected, e.g., intracranial meningioma or other brain tumor; (7) Control of the episodes with anticonvulsive medication and failure of control with other therapeutic measures, e.g., anticoagulants; and (8) the absence of the other conditions that can account for transient attacks of focal weakness, viz., migraine, transient cerebral ischemia associated with thrombosis, and thrombocythemia. In the cases to be presented, these several criteria are not represented with any uniformity.

It is not our intention to discuss the mechanism of the paralysis. This has been done by Efron (1961). Paralysis is commonly attributed to inhibition rather than exhaustion or anoxia. The train of events is in keeping with the concept of inhibition. The presence of paresthesias may indicate that an acute disturbance in the cortical sensory apparatus may temporarily preclude movement.

\section{THE PATIENTS}

Case 1: A man, aged 53, reported 8 spells in 3 months in which a warm sensation like sunlight ascended on the left side from the inguinal region to the lower rib margin following which he felt giddy and had to lie down. Although no convulsive movements occurred, the left leg became paralyzed or weak for about 2 minutes and could be moved only but passively lifting it. An EEG was normal. Ischemia in the territory of the right anterior cerebral artery was suspected but 10 weeks later twitching of the leg was added to the clinical picture and the patient proved to have a glioblastoma.

Case 2: A man, aged 35, for two years had frequent spells in which he fought for breath, felt lightheaded and for about 2 minutes was unable to move the left leg from the hip down. To cross his knees he had to lift the leg with his hands. There was no convulsive movement. At the same time, coordination was lost in the left fingers and hand and he would drop a cigarette. After two years he began to have a buzzing sensation in the sole of the left foot at the beginning of a spell and later a rhythmic pulsation in the left gluteal region. Although sometimes faint, he had not lost consciousness. An EEG was normal. Carotid angiography, which at first had been normal later disclosed a tumor which at operation was an astrocytoma.

Case 3: A woman, aged 59, suddenly felt a sharp pain in the head, became dizzy, leaned against a door, may have lost consciousness briefly, and fell to the floor unable to move the right leg. She crawled to a telephone and called a neighbor saying she thought she had a stroke. She was unable to move the leg for 15 minutes. Four weeks later while having a radioactive brain scan she felt faint and examination disclosed moderate weakness of the right lower extremity and slight weakness of the right upper extremity, although her husband who 
had been with her constantly had seen no twitching. Angiography showed the pattern of a glioblastoma and the diagnosis was confirmed at autopsy one year later.

Case 4: A right-handed woman, aged 72 , had four identical spells, three, three years before and one on the day of admission. In each, first the right hand and arm became numb and weak and things were dropped. Almost immediately "something" went up the right arm to the right side of the face and jaw, speech became slow and thick and the patient used wrong words. Once she could not give her name. Recovery was gradual and in about 30 minutes was complete. The patient had noted no twitching of the fingers or face and her husband who witnessed two spells saw none. There was no headache. Neurological examination was negative. An EEG showed bilateral bursts of theta waves in the temporal regions bilaterally, more prominent on the left. Transient ischemia associated with cerebrovascular disease was suspected but a left carotid angiogram showed a $5 \mathrm{~cm}$. calcified meningioma in the left posterior frontal region. The arterial tree was normal and no region of constriction of vessels by tumor was evident.

In these four tumor cases the evidence for non-convulsive seizure paralysis is probably strongest. The possibility exists that brief minor twitching was overlooked. In three of the four cases, TIAs were suspected at first while in the fourth the patient was thought to be "hysterical".

In the following six cases, the patients are presumed to have had vascular disease as the underlying process. The possibility of ictal paralysis was entertained, but usually not convincingly distinguished from ischemia, migraine, or post-ictal deficit.

Case 5: A man, aged 36, with severe hypertension and a history of 5 small strokes in the previous 7 months, while in the hospital began to have focal seizures affecting the left lower extremity. On one occasion immediately after the patient had been examined and power in the left toes and hip had been found to be good, he reported that the left foot felt warm, hot or burning, just as in previous seizures. The sensation persisted for the next 5 minutes during which time it was found that the patient was unable to move any part of the lower extremity, although there was no twitching. The limb was flaccid. At the end of 5 minutes the second toe began to flex rhythmically following which all the toes, then the ankle and knee began to jerk regularly in flexion at 2 per second. This continued for 3 minutes and then stopped suddenly. The patient was able to flex the left hip after one minute and the toes after 30 minutes. He remained lucid and cooperative. There was no change in facial color. Numbness had been limited to the foot. An EEG during a focal convulsive seizure showed paroxysmal slow wave activity over the right hemisphere.

Case 6: A hypertensive woman, aged 77, was admitted to the hospital because of acute episodes of numbness and dysarthria lasting 20 to 30 minutes. Seven years before, she had had a right-sided stroke interpreted as an intracerebral hemorrhage because of blood in the cerebrospinal fluid (CSF). During convalescence from the stroke one convulsive seizure occurred and diphenylhydantoin was begun and used for about 6 years. Recovery from the stroke was complete except for slightly decreased dexterity of the right hand and a right Babinski sign. The patient was well until the day of admission when she had the first spell of numbness of the right hand and forearm and dysarthria. A second similar episode occurred three days later and a left carotid angiogram, carried out immediately, was normal. About 10 minutes after the last injection of contrast material a third episode occurred and 25 minutes later a fourth, both of these spells being witnessed. First the patient complained of numbness of the 2nd, 3rd, and 4th fingers of the right hand, followed by numbness of the right forearm. About 7 minutes later dysarthria was evident and at the same time a severe right central facial weakness appeared. In about 15 minutes restoration was complete. At the height of the episode the patient was alert and able to follow commands and do simple arithmetic. The right leg was not involved. The blood pressure was 190/100. At no time was there twitching of the face or fingers. A cerebral CT scan showed an area of very low density several $\mathrm{cm}$. in extent in the left inferior pre-central region (Broca's area) indicative of an old infarct. An EEG showed delta slowing with some sharp waves in the left temporal area. Diphenylhydantoin was prescribed again and in the following days numbness of the right arm recurred which spread to the right shoulder and right mastoid region. Speech was involved only once. When diphenylhydantoin was increased to 300 $\mathrm{mg}$. daily the spells stopped.

\section{Comment:}

In the angiogram there was no evidence of arterial stenosis or source of embolic material. The delay between numbness of the arm and the onset of dysarthria was atypical for ischemia and more characteristic of a seizure or migraine. The cessation of spells after increased diphenylhydantoin supports a seizure mechanism. Possibly transient ischemic attacks based on circulatory factors can occur at sites of old infarction in the absence of detectable arterial stenosis.

Case 7: A normotensive, obese, moderately severe diabetic woman, aged 64 , suddenly developed numbness of the right side of her face and lips, slurring of speech, difficulty in finding correct words and weakness of the right side of the face, all disappearing in about five hours. She was well until 9 months later when an identical attack cleared in about 30 minutes. Another episode 4 days later resulted in her admission to the hospital. No twitching was noted by the patient or her family. The neurological examination was normal. A CT scan five days after admission showed a circumscribed quadrilateral lesion of very low density in the region of Broca's area. It represented a region of old infarction about $2.5 \mathrm{~cm}$ in extent. A left carotid angiogram showed a $2 \mathrm{~cm}$. long irregular narrowing of the internal carotid sinus with reduction of the lumen to about $3.5 \mathrm{~mm}$. There was no intimal ulceration. The intracranial vasculature was normal. There was a systolic aortic murmur consistent with aortic valve disease. Cardiac fluoroscopy showed changes consistent with either a large ventricular scar or aneurysm. Successive electrocardiograms raised the suspicion of the sick-sinus syndrome. An EEG showed left frontotemporal slowing.

In summary, two similar episodes were associated with an old infarct in Broca's area. Could recurrent emboli, for example, from the internal carotid artery, produce identical transient deficits in a region already the site of destruction? The situation was similar to that described in Case 6, in which a scar was associated with recurrent spells of weakness and numbness. It is postulated that the original espisode 9 months before was the result of an embolus either from the internal carotid artery or the heart.

Case 8: A man, aged 77, with atrial fibrillation gave a history that for several years he failed to see clearly the left hand object of a horizontally arranged series. One afternoon while reading aloud he stopped and complained of greater difficulty seeing the left hand side of the page. A moderately severe headache appeared. At dinner he ate less neatly than usual. Later, on getting out of a cab he slammed the door on his wife who was standing on his left. When walking about he seemed not to see properly and had to hold on to things. At the opera that evening he had difficulty reading the program. At 8:30 p.m. he suddenly stiffened in his seat, turned his head 
to the left, lost consciousness and began a rhythmic jerking of the limbs lasting about 1 minute. He was taken to the hospital, given anticonvulsant therapy, and within one hour began to talk a little and in 24 hours was back to his usual state except for visual hallucinations, illusions and distortions, and slight signs of a non-dominant parieto-occipital deficit. The CSF was clear. The blood pressure was 160/100. An EEG 4 days after the ictus showed occasional $2-4 / \mathrm{sec}$. slow waves mainly in the left frontal region. Recovery was complete. The diagnosis lay between cerebral embolism and arterial stenosis in the left middle cerebral artery territory. Nineteen months later one afternoon again while reading aloud the patient complained of visual difficulty with the left side of the page and was noticed not to be using his left hand properly. A headache of moderate severity had appeared. At supper the patient handled his fork poorly with the left hand and afterwards dragged the left leg and slurred his speech. Slowly during the next two hours he developed total paralysis of the left arm and leg. His son, a physician, was with the patient the entire time and noted no twitching. Examination in the emergency department at 9 p.m. disclosed a left hemiplegia, left homonymous field defect and unawareness of the left-sided deficit. While being examined, partial continual epilepsy was noted beginning in the left corner of the mouth and extending to the left side of the neck. After one-half hour the fingers of the left hand became involved. An hour later partial continual epilepsy involved the left shoulder, trunk, left foot and thigh, but spared the left face and hand. At 11 p.m. while being examined the patient had a two-minute generalized seizure with rhythmic adduction of the legs, quivering of the body, deviation of the eyes to the left, apnea and bilateral jerking of the limbs. Within three hours, the left limbs had recovered to about $7 / 10$ strength. Recovery was almost complete in 10 days, but during this period there were continual visual and auditory hallucinations. An EEG 4 days from the onset showed bilateral delta activity in both temporal regions, more on the left. The CSF was clear. Angiography was not carried out. The patient began diphenylhydantoin therapy and during the next 9 years there was no recurrence. He died from an acute myocardial infarction and at autopsy no obvious surface abnormality of the brain was noted.

\section{Comment:}

The two episodes had a similar onset and after a delay of several hours during which a neurologic deficit was present, a generalized seizure occurred. It is possible that in the second episode another embolus was carried to the right middle cerebral artery but against this is the slow and gradual development of the left hemiplegia. Both spells would be atypical for transient ischemic attacks arising in the middle cerebral artery territory on the basis of stenosis of the middle cerebral stem, in that frank clonic seizure activity almost never occurs as a part of a transient ischemic attack. A seizure usually indicates another process. Atypical latelife migraine should be considered since a headache accompanied each spell and paralysis in the second spell developed gradually as it frequently does in migraine. However, a convulsive seizure is extremely uncommon in accompanied migraine. Focal cerebral seizures of inobvious cause are recognized as occurring in patients with atrial fibrillation probably on the basis of otherwise asymptomatic cerebral embolism.

Case 9: A woman, aged 64, with atrial fibrillation suddenly began to "talk gibberish" but had no paralysis of the limbs. Recovery was not quite perfect, and at the end of two months slight difficulty in comprehension, reading and writing remained. A diagnosis of cerebral embolism was made. Two years later the patient's husband found her at 4:00 a.m. fumbling for something on her bedside table. She kept her eyes closed and speech was more impaired than usual. In the next 2 hours the patient slowly and progressively developed speechlessness and complete paralysis of the right side, face, arm and leg. There was no twitching. The limbs began to move in a few hours and in 5 days the patient had in every way returned to her previous level of functioning. Long term anticoagulant therapy was begun. Nine months after the second episode the patient suffered another spell in which she first called to her husband, "I don't know how to let the water out of the sink." Also, she did not see her false teeth which were lying directly in front of her. In the next 15 minutes she gradually sank into stupor with severe impairment of speech and weakness of the right side. Again there was no convulsion. In four hours she began to move the limbs and in 24 hours she was back to her former level. The central retinal artery pressure was 80 bilaterally and there were no bruits in the neck. Angiograms were not made. An EEG showed a left temporo-occipital focus of sharp waves. On diphenylhydantoin therapy the patient had no further spells in five years.

\section{Comment:}

In this case the diagnosis of seizures is a tentative proposal to explain recurrent spells not obviously of another cause. The diagnosis of seizures was supported by the cessation of spells on anticonvulsant therapy. The gradual development of the neurologic deficit and the ineffectiveness of anticoagulants are against the diagnosis of recurrent ischemia due to embolism, stenosis or thrombosis of the middle cerebral artery. Angiography would have been helpful. Migraine can be associated with a temporary paralytic accompaniment even in the absence of a personal or family history of ordinary migrainous phenomena. The case resembles Case 8.

Case 10: Six years before admission a hypertensive man, aged 78 , with atrial fibrillation suddenly developed a left homonymous hemianopia with a fixed quadrantanopia thereafter. Two years before admission he had about 20 episodes of numbness of the face, arm and leg on the left side, lasting up to one hour and ceasing in about 17 days. The blood pressure was 170/100. Carotid and vertebral angiography was normal except for a questionable attenuation of the posterior branches of the right middle cerebral artery. The right posterior cerebral artery filled well. Four days before admission the patient began to have spells lasting about 30 minutes in which he experienced numbness of the medial three fingers of the left hand and forearm, with difficulty lifting a utensil, a tight feeling around the mouth, and dysarthria. While in the hospital, in a period of 18 days he had approximately 20 further spells during several of which examination showed marked weakness of the extensors of the left wrist, slightly reduced sensation in the left forearm, dysarthria, and severe weakness of the left side of the face. In no spell was twitching or jerking of the fingers or face observed. The episodes evolved over a period of several minutes with a gradually spreading numbness of the left arm followed by perioral numbness. The manifestations took up to one hour to disappear. In one spell there was sparkling in the right visual field. While the patient was receiving diphenylhydantoin therapy, the spells were limited to perioral numbness. Four spells occurred while the patient was receiving heparin intravenously. One spell occurred during normal cardiac monitoring with the Holter instrument. Intravenous diazepam failed to alter the course of one spell. An electroencephalogram between spells showed delta slowing in the right mid-temporal area. 
Right carotid angiography again showed no arterial stenosis or source for embolism. There was no retrograde arterial filling across borderzones. A computerized tomographic X-ray (CT) scan of the head was consistent with an old infarct in the territory of the right posterior cerebral artery. Sodium warfarin therapy was undertaken for one year during which period there were no further spells. In the next four months without anticoagulants there also were no spells.

\section{Comment:}

Only the attacks occurring during the last admission are referred to here. The evidence for nonconvulsive seizure activity is not strong but diphenylhydantoin therapy did have a beneficial effect. The gradual development of symptoms in each episode is unlike ischemia and suggests a seizure or migrainous process. An EEG showed an abnormality which could be related, however, to an old posterior cerebral artery infarction. An EEG was not obtained during an attack. If the spells were seizures, what was their etiology? As already mentioned, atrial fibrillation occasionally is associated with minor asymptomatic cerebral infarcts which then serve as the focus for seizure activity.

Regarding a circulatory disturbance as possibly the cause of the spells, the combination of sensory-motor manifestations involving the face and fingers on one side, indicated ischemia of the superficial cerebrum rather than of the deep or penetrating artery territory. Yet, on angiography, there was no stenosis of the internal carotid or middle cerebral artery or their terminal branches. The arterial branch whose involvement would be required to account for the symptoms was of large enough caliber to be visualized angiographically. The spells continued to occur during intravenous heparin therapy and lasted up to 1 hour, not a few seconds or minutes as do most transient ischemic attacks. Although the episodes ceased during long term sodium warfarin therapy, they did not recur on cessation of therapy. There is the outside possibility that the spells arose in the thalamo-capsular region on the basis of hypertensive small-vessel vascular disease, but the highly focal nature of the neurologic deficit is against this. The concept of vasospasm is rarely invoked as a cause of ischemia nowadays.

In this case as well as some of the others, the main alternative diagnosis to seizure is a migrainous process. As mentioned above, late-life migraine can cause paresis or paralysis and the diagnosis is facilitated when there also are visual symptoms or an accompanying sensory march. In the absence of visual phenomena, interpretation is uncertain although an unequivocal sensory march is still strong evidence. Headache is important if present, but often it is absent. In the present case there were sensory symptoms and the syndrome evolved slowly, both characteristic of migraine. The duration of the transient spells is more in keeping with migraine or seizure than the ischemia of vascular disease. The occurrence of migrainous paralysis for the first time at the age of 78 must be exceptional.

Case 11: A woman, aged 63, who was slightly vague and forgetful was admitted to the hospital because of right-sided weakness and speechlessness which had developed in a period of about 4 hours. She was receiving sodium warfarin therapy because of three spells of gradually developing unresponsiveness and inability to use the left leg which had occurred one year before. On examination in the emergency department there was a right hemiparesis and jargon aphasia. There was a severe right facial weakness and an absent grip on the right. Dramatic recovery occurred in about 14 hours. A left carotid arteriogram was normal. A right carotid arteriogram showed a persistent trigeminal artery supplying the upper basilar territory. Both vertebral arteries and the lower basilar artery were small. No source of embolus was found. In the two months after discharge, she had three further spells of right-sided weakness and inability to speak, lasting about 24 hours. These also occurred while on sodium warfarin therapy. An EEG showed scattered 3 and 4 per second slow waves, more on the left side. Five months after discharge she had two minor spells of slurred speech and drowsiness clearing in about two hours. Six months after discharge, one morning the patient developed a terrible headache, sleepiness, and staggering gait. Speech was slurred and the patient gradually became stuporous. On readmission to the hospital she lay immobile with eyes closed. Examination disclosed a complete left hemiplegia with corresponding sensory loss and bilateral Babinski signs. In
24 hours, the left-sided deficit had largely disappeared. During this admission one day, the left forearm, arm and toes jerked irregularly at about twice per second with an amplitude of 2 to $3 \mathrm{~mm}$. The right thumb did likewise. The jerking was not associated with weakness. Six months later, she had another spell of right-sided weakness clearing in one hour. During the period of two years in which the above spells occurred, the patient was developing a severe chronic frontal lobe deficit (abulia, psychomotor retardation) combined with poor memory. In the following year, episodes of left-side weakness, dysarthria, and incontinence occurred but were of decreasing severity. The patient became more and more inactive and died from acute myocardial infarction in a demented state at the age of 68 , five years after the onset of the illness. Neuropathological examination showed advanced brain atrophy and the microscopic changes of Alzheimer's disease. In addition there was a $3 \times 1 \mathrm{~cm}$. area of old infarction in the inferior parietal lobe on the left side.

\section{Comment:}

No satisfactory explanation for the attacks of paralysis was forthcoming despite extensive clinical and laboratory studies. It is possible that the spells were due to lower basilar ischemia, but dizziness, diplopia, and nystagmus were never present. On the other hand, aphasia and hemianopia occurred. Someone attended her at home at all times and no seizure activity was described. In the hospital, there was minor twitching on one occasion but it was not rhythmic. However, it may justify including the patient in this group of cases. The speed of recovery from a severe deficit was remarkable.

\section{ICTAL DEFICITS OTHER THAN PARALYSIS}

The occurrence of paralysis without convulsions prompted the question whether seizure activity in cerebral regions other than motor can take the form of sudden deficits in the absence of classical auras. It is well recognized that epileptic discharge in the Rolandic or supplementary motor area of either hemisphere or in the posterior Sylvian gyri of the dominant hemisphere may cause arrest of speech, dysarthria, or dysphasia, without convulsive movement (Penfield and Roberts, 1959). Gowers (1885) referred to a patient 
who saw colors, especially green and blue, in slight attacks while severe fits were preceded by simple loss of sight. Holmes (1927) pointed out that in focal sensory epilepsy the attack may occasionally take the form of a sudden temporary loss of sensation or in visual epilepsy a sudden hemianopia without preceding scintillations. It has been suggested that transient global amnesia is a seizure phenomenon (Fisher and Adams, 1964).

\section{The Hypertensive}

Amaurosis-Seizure Syndrome

In this remarkable and puzzling syndrome associated particularly with hypertensive encephalopathy of renal origin, central blindness precedes the onset of generalized seizures. The following two cases are examples.

Case 12: A woman, aged 26, had lupus erythematosis for 10 years marked by arthritis, skin rash, nephrotic syndrome, moderate ascites, urea nitrogen $116 \mathrm{mg} . \%$, anemia (hematocrit $31 \%$ ), thrombocytopenia $(170,000$ per $\mathrm{cu} \mathrm{mm}$.) and hypoproteinemia $(5.1 \mathrm{gm} . \%)$. The blood pressure, which had usually been normal, rose in a period of two weeks to a range of $150 / 100$ to $160 / 110$. After having had a headache for a day, the patient awakened at 4:00 a.m. unable to see. "Everything looked blank." The patient was clear mentally, although alarmed. At 8 a.m. she opened her eyes when shaken but did not carry out commands or talk. At 9:15 a.m. she had a generalized seizure followed by stupor. Further seizures occurred at 11 a.m., 2:25 p.m., and 2:35 p.m. and the patient remained in deep coma. The seizures were controlled with intravenous diazepam. There was no papilledema. The CSF was clear. Over a period of four weeks, the patient gradually recovered completely except for a left homonymous hemianopia which disappeared in the following several months.

Case 13: A man, aged 28, with chronic glomerulonephritis and renal failure entered the hospital because of a generalized convulsive seizure with urinary incontinence. Eight hours before the seizure, he had suddenly noted dimness of vision and spots before the eyes. On admission he was stuporous. There was early papilledema. The blood pressure was $235 / 135$. In a few hours the patient temporarily regained his faculties. The BUN was $216 \mathrm{mg} . \%$ and hematocrit $29 \%$. The CSF was under a pressure of $190 \mathrm{~mm}$. and contained 200 red blood cells per cubic mm. At autopsy two weeks later, the only gross abnormality of the brain was slight cerebral edema.

\section{Comment:}

Jellinek et al. (1964) presented four cases and Evans (1933) six cases in which visual symptoms were closely linked to convulsions. Blindness is usually attributed to vasoconstriction of the posterior cerebral arteries, with the production of cortical ischemia. Edema and the rapid return of vision once the blood pressure is lowered supports this theory. However, in thrombosis or embolism of the posterior cerebral artery, seizures are rare. Also, the visual accompaniments of migraine, including bihemianopic blindness, although theoretically vasospastic in origin, are almost never associated with seizures, at least as commonly recognized. Furthermore, the acute encephalopathy associated with severe essential hypertension is not often marked by blindness. The vasoconstriction of the hypertensive amaurotic-seizure syndrome would have to be a unique process that produces not only a calcarine cortical disturbance and blindness but also presumably brain edema that is particularly epileptogenic, i.e., two different processes would be required.

In the remarkable case of permanent loss of peripheral vision during acute nephritis and convulsions reported by Bramwell et al. (1915), the cerebral lesions which were ischemic lay for the most part in the lateral occipital region bilaterally in the most distal middle cerebral distribution, and were possibly the result of hypotension or hypoxia during the seizures rather than representing the primary hypertensive visual disturbance.

The regularity with which seizures follow visual symptoms in these cases is reason enough to examine the possibility that the blindness is the result of non-convulsive seizure activity. This would have the advantage that only one process would have to be invoked. In the cases of Jellinek et al. (1964) in the acute stage during the period of blindness, the EEG showed bilateral synchronous, often rhythmic, occipital sharp and slow activity and loss or impairment of alpha rhythm. While seizure activity might provoke an ambylopia lasting for up to several hours before the onset of convulsions, it would hardly explain Case 3 of Jellinek et al. in which the visual deficit persisted for 10 days despite anticonvulsant therapy and cleared promptly with antihypertensive therapy. However, in that case the neurologic state was complex.

\section{DISCUSSION}

Although the cases were discussed above as possible examples of nonconvulsive seizure paralysis, they may well be heterogeneous with more than one mechanism. For example, the two patients with paralysis preceding a convulsive seizure (Cases 5 and 8 ) should be kept apart from the others. In the remainder, no twitching was observed in relation to attacks of paralysis. The cases of tumor (Cases 1 to 4) appear to have more in common with each other than with the vascular cases (Cases 5 to 10). It is possible that in the vascular cases, angiography failed to portray symptomatic arterial stenosis at sites of old strokes. If conclusions were to be based only on hard facts it would be accurate to regard the attacks in these patients as a series of puzzling events without making a final commitment to a precise diagnosis or pathogenesis. Indeed, the mechanism in some cases may be one that is not yet recognized.

Transient paralysis involved the face in two cases, face and arm in two, leg alone in four and face, arm and leg in three. The patient remained alert in eight cases and became sleepy or faint in the other three. The duration of symptoms was 2 minutes in two cases, 5 to 30 minutes in five, 1 hour in one, 3 hours in one and 1 day in two cases. The slow gradual onset of the paralysis over a period of hours in Cases 8 and 9 was remarkable. In none of the vascular cases did the paralytic attack end in a persistent stroke. Of the 11 cases, seven reported sensory phenomena - numbness, thermal dysesthesias or visual disturbance - in association with the paralysis but this does not assist in the interpretation since migraine, TIA, and seizure may each have a combination of motor and sensory symptoms. It would seem that a requisite for the phenomenon in most cases is the existence of a focus of brain damage due to tumor or infarct. 
Regarding management, a reliable description of the attack is a prime requisite in diagnosis. A CT scan will disclose a tumor or old infarct. Angiography will usually be necessary and will rule out arterial stenosis as the cause. Once tumor is excluded a trial of anti-seizure medication is warranted.

\section{REFERENCES}

BRAMWELL, B., BOLTON, J. S. and ROBINSON, W. (1915). Bilateral lesions of occipital lobes with retention of macular as distinct from panoramic vision. Brain, 38, 447-472.

COLLIER, J. (1928). Lumleian lectures on epilepsy. Lancet 1,687-692.

DUTIL, M. A. (1883). Des Paralysies postepileptoides transitoires. Rev. med., 3, 161185.
EFRON, R. (1961). Post-epileptic paralysis: Theoretical critique and report of a case. Brain, 84, 381-394.

EVANS, H. (1933). Hypertensive encephalopathy in nephritis. Lancet, 2,583 588.

FISHER, C. M. and ADAMS, R. D. (1964). Transient global amnesia. Acta Neurological Scandinavica, Suppl. 9, 40, 1-83.

GASTAUT, H. (1970). Clinical and electrophysiological classification of epileptic seizures. Epilepsia, 11, 102-113.

GOWERS, W. R. (1885). Epilepsy and Other Chronic Convulsive Diseases. William Wood and Company, New York.

HOLMES, G. (1927). Local epilsepsy. Lancet, 1, 957-962.

JACKSON, J. H. (1890). The Lumleian lectures on convulsive seizures. Brit. M. J., 1, 821827.
JELLINEK, E. H., PAINTER, M., PRINEAS, J. and RUSSELL, R. R. (1964). Hypertensive encephalopathy with cortical disorders of vision. Quart. J. Med., 33, 239-256.

KOFMAN, O. and TASKER, R. (1967). lp silateral and focal inhibitory seizures. Neurology, 17, 1082-1086.

LENNOX, W. G. (1960). Epilepsy and Related Disorders. Little, Brown and Company. Boston. Vol. 1, p. 210.

PENFIELD, W. G. and JASPER, H. H. (1954). Epilepsy and the Functional Anatomy of the Human Brain. Little, Brown and Company, Boston:

PENFIELD, W., and ROBERTS, L. (1959). Speech and Brain Mechanisms. Princeton University Press, Princeton, New Jersey.

RASMUSSEN, T. (1974). In Handbook of Clinical Neurology. Vinken, P. J. and Bruyn, G. W. North-Holland Publishing Company, Amsterdam. Vol. 15, p. 80. 